PharmacoEconomics \& Outcomes News 852, p3 - 2 May 2020

\title{
ICER releases evidence report on elexacaftor/tezacaftor/ivacaftor for cystic fibrosis
}

A newly released evidence report from the Institute for Clinical and Economic Review (ICER) on the comparative clinical effectiveness and value of elexacaftor/tezacaftor/ivacaftor [Trikafta; Vertex Pharmaceuticals] for patients with cystic fibrosis provides high confidence of substantial net health benefits but notes that the prices "are out of proportion" to these benefits.

The ICER awarded its highest evidence rating (A) to elexacaftor/tezacaftor/ivacaftor, a cystic fibrosis transmembrane conductance regulator (CFTR) modulator, with evidence showing a high certainty of substantial net health benefits compared with tezacaftor/ivacaftor [Symdeko; Vertex] or standard care for the indicated populations where clinical data was available. The ICER also determined that use of elexacaftor/tezacaftor/ivacaftor in the subgroup of patients who are heterozygous for the F508del mutation and a residual function mutation is "likely to be at least as good as" and "possibly better" than treatment with tezacaftor/ivacaftor. New data examined by the ICER on three other CFTR modulators, ivacaftor [Kalydeco; Vertex], lumacaftor/ivacaftor [Orkambi; Vertex] and tezacaftor/ivacaftor, confirmed previous evidence ratings, indicating a high certainty of substantial net health benefit with ivacaftor, small net health benefit with potential for substantial benefit with tezacaftor/ivacaftor, and small net health benefit with lumacaftor/ ivacaftor, compared with best supportive care. The updated Evidence Report incorporated changed made following comments received during the 5 -week comment period for the draft version.

The 8-month review also considered contextual considerations and potential other benefits, with patients and caregivers sharing personal stories describing the negative impact of cystic fibrosis on their lives and the positive impact offered by the four CFTR modulators.

The ICER recommended the health-benefit price benchmark for elexacaftor/tezacaftor/ivacaftor be set at \$US67 900-\$85 500 per year, which would require the current list price of the treatment to be discounted by at least $73 \%$. ICER also announced an indefinite postponement of their cystic fibrosis public meeting due to the current public health concerns around meetings and travel due to COVID-19.

"Trikafta is likely to alter the course of disease for the majority of patients with CF [cystic fibrosis], transforming their lives and the lives of caregivers. It is critical that all eligible patients be able to access CFTR modulators", said David Rind, ICER's Chief Medical Officer. "Despite being transformative therapies, the prices set by the manufacturer costing many millions of dollars over the lifetime of an average patient - are out of proportion to their substantial benefits. When a manufacturer has a monopoly on treatments and is aware that insurers will be unable to refuse coverage, the lack of usual counterbalancing forces can lead to excessive prices".

Institute for Clinical and Economic Review. ICER Releases Evidence Report on Treatments for Cystic Fibrosis. Internet Document : 27 Apr 2020 . Available from: URL: https://icer-review.org/announcements/cf_evidence_report_2020/ 